\title{
Clinical significance of Smac and survivin expression in breast cancer patients treated with anthracycline-based neoadjuvant chemotherapy
}

\author{
YING-CHUN ZHAO $^{1^{*}}$, YAN WANG $^{2^{*}}$, XIAO-JIAN NI ${ }^{3 *}$, YONG LI $^{4}$, \\ XIU-MING WANG ${ }^{1}$, YONG-YUN ZHU ${ }^{1}$ and CHUAN-YU LUO ${ }^{1}$
}

\begin{abstract}
${ }^{1}$ Department of Breast Surgery, The Second People's Hospital of Wuhu Affiliated with Wannan Medical College;
${ }^{2}$ Department of Plastic Surgery, Yijishan Hospital Affiliated with Wannan Medical College, Wuhu, Anhui 241000;

${ }^{3}$ Department of General Surgery, The First Affiliated Hospital of Nanjing Medical University, Nanjing, Jiangsu 210029;

${ }^{4}$ Department of Pathology, The Second People's Hospital of Wuhu Affiliated with Wannan Medical College, Wuhu, Anhui 241000, P.R. China
\end{abstract}

Received June 11, 2013; Accepted November 25, 2013

DOI: $10.3892 / \mathrm{mmr} .2013 .1842$

\begin{abstract}
The second mitochondria-derived activator of caspases (Smac), an antagonist of the inhibitor of apoptosis protein (IAP), increases chemosensitivity in vitro. Survivin, an IAP family member, mediates cancer cell survival and chemoresistance. The present study investigated the correlation between Smac and survivin expression in primary breast cancer, and the sensitivity to anthracycline during neoadjuvant chemotherapy (NAC). Pre-treatment biopsies and post-anthracycline treatment tumor sections were analyzed from 98 cases. Biomarker expression was evaluated by immunohistochemistry in tumor samples from clinical stage II and III anthracycline-based NAC-treated breast cancer. A univariate analysis indicated that the estrogen receptor (ER), Smac and survivin were significantly predictive of a pathological complete response (pCR) $(\mathrm{P}=0.004$, 0.001 and 0.037 , respectively) in pre-chemotherapy samples. ER, Smac and survivin expression was also significant for pCR on the multivariate analysis $(\mathrm{P}=0.001,0.031$ and 0.012 , respectively). An inverse association was identified between survivin and Smac expression ( $\mathrm{r}=-0.217, \mathrm{P}=0.032$; and $\mathrm{r}=-0.335, \mathrm{P}=0.003$, respectively) prior to and following NAC. The patients with low survivin expression or high Smac expression had significantly longer disease-free survival
\end{abstract}

Correspondence to: Professor Chuan-Yu Luo, Department of Breast Surgery, The Second People's Hospital of Wuhu Affiliated with Wannan Medical College, 231 Jiuhuashan Road, Wuhu, Anhui 241000, P.R. China

E-mail: 1cy0508@hotmail.com

*Contributed equally

Key words: breast cancer, chemotherapy sensitivity, second mitochondria-derived activator of caspases, survivin
(DFS; $\mathrm{P}=0.012$ and $\mathrm{P}=0.020$, respectively) and overall survival ( $\mathrm{OS} ; \mathrm{P}=0.01$ and $\mathrm{P}=0.033$, respectively) compared with the patients with high survivin or low Smac expression. Cox regression analyses demonstrated that survivin, Smac and clinical stage were independent predictors for DFS and OS. The present study indicated the significance of Smac and survivin in determining the breast cancer response to anthracycline-based chemotherapy, and may permit further stratifying of pre-chemotherapy patients to undertake more tailored treatments.

\section{Introduction}

The use of neoadjuvant chemotherapy (NAC) or pre-operative systemic therapy is being increasingly considered for patients with operable breast cancer (1), since the survival rates are similar to those in patients receiving standard post-operative chemotherapy and the success of breast-conserving surgery is significantly improved in patients treated with NAC. The aim of NAC is to achieve a pathological complete response (pCR), i.e., the absence of malignant cells at the tumor site, as pCR has been associated with longer disease-free survival (DFS) and overall survival (OS) rates (2). However, a small proportion of patients are likely to fail to respond or their condition will progress during primary chemotherapy. The identification of such non-responding patients at an early stage may spare these individuals from undertaking the toxicity of ineffective chemotherapy and allow them to begin non-cross resistant regimes or alternative treatment strategies. The identification of tumor biological markers that accurately predict the response to treatment may aid in optimizing NAC.

Second mitochondria-derived activator of caspases (Smac) is a novel pro-apoptotic protein, also known as direct inhibitor of apoptosis-binding protein with low pI (DIABLO), which was initially identified independently by two groups in $2000(3,4)$. SMAC/DIABLO is released from mitochondria into the cytosol concurrently with cytochrome $c$ and eliminates the inhibitory effects of the inhibitor of apopotosis 
proteins (IAPs) to promote apoptosis. SMAC/DIABLO has a significant regulatory role in the sensitization of cancer cells to immune- and drug-induced apoptosis (5). As a member of the IAP family, survivin has been demonstrated to be involved in apoptosis inhibition and cell cycle control. The formation of the survivin-SMAC complex requires the N-terminus of mature SMAC/DIABLO, and the distribution of survivin and SMAC/DIABLO within the cell reveal that they co-localize within the cytosol during interphase. Thus, SMAC/DIABLO promotes apoptosis by interacting with survivin (6).

However, there are a number of studies concerning the clinical values of Smac and survivin expression in advanced breast cancer patients treated with NAC. To aid in our understanding of the correlation between the expression of survivin and Smac, and the clinical outcome of patients with advanced breast cancer receiving NAC, survivin and Smac expression was measured in the present study using immunohistochemistry prior to and following NAC treatment in breast cancer tissue specimens. The expression levels were analyzed for the correlation with clinicopathological factors, the response to neoadjuvant chemotherapy and the survival rate.

\section{Patients and methods}

Patients. The consecutive cohort comprised 98 patients who presented with locally advanced primary breast cancer between December 2005 and December 2009 at the Department of Breast Surgery, Wuhu Second People's Hospital, Wannan Medical College (Wuhu, China) and were treated with anthracycline-based NAC. The Research Ethics Committee of Wuhu Second People's Hospital affiliated to Wannan Medical College approved the study. The median age of the patients was 49 years old (range, 32-69 years). The patients were staged according to the Union Internationale Contre le Cancer tumor node metastasis (TNM) classification (7), and a core biopsy was performed prior to chemotherapy to allow pathological diagnosis and evaluation of biological parameters. The patients were then treated with four cycles of anthracycline-based therapy $\left[500 \mathrm{mg} / \mathrm{m}^{2} 5\right.$-fluorouracil (5-FU), 75-100 mg/m² epirubicin and $500 \mathrm{mg} / \mathrm{m}^{2}$ cyclophosphamide on day one of a 21-day cycle]. No further adjuvant chemotherapy was allowed in the patients included in the present study and no patient received neoadjuvant or adjuvant trastuzumab. The patients then underwent breast-conserving surgery or mastectomy within 3-4 weeks of the final treatment, and tumor tissues were obtained at surgery. Written informed consent was obtained from all patients. Patients with metastatic carcinoma to four or more axillary lymph nodes or with breast-conserving surgery, received radiotherapy. All the estrogen/progesterone receptor (ER/PR)-positive cases received adjuvant hormonal treatment. A histological examination was used to confirm invasive carcinoma by needle biopsy. The patients were followed up for 10-68 months (median, 57 months) during which, 28 patients (28.6\%) succumbed to the tumor and 10 (10.2\%) developed local or distant metastasis.

Evaluation of treatment response. An assessment of the tumor response was evaluated prior to chemotherapy and following each cycle. A pathological response to NAC was evaluated using histological examination of the surgical specimens.
The absence of invasive tumor in the final surgical breast and axillary lymph node samples was defined as pCR, while other conditions were regarded as residual disease (RD) (8).

Immunohistochemistry. Immunohistochemistry was performed on core biopsies and whole tumor sections. Formalin-fixed paraffin sections of tumor tissues were subjected to immunohistochemical staining. The sections were cleared, treated with $0.3 \% \mathrm{H}_{2} \mathrm{O}_{2}$ for $10 \mathrm{~min}$ at room temperature, placed in $0.01 \mathrm{mmol} / 1$ sodium citrate $(\mathrm{pH} 6.0)$ and heated in a microwave oven for antigen retrieval. The slides were incubated with rabbit polyclonal anti-Smac antibodies (1:200; Santa Cruz Biotechnology Inc., Santa Cruz, CA, USA) or rabbit polyclonal anti-survivin antibodies (1:100: Santa Cruz Biotechnology Inc.) at $4^{\circ} \mathrm{C}$ overnight in a humidified atmosphere, rinsed three times in $0.1 \mathrm{mmol} / 1 \mathrm{PBS}$ for $2 \mathrm{~min}$, incubated for $30 \mathrm{~min}$ at room temperature with goat anti-mouse horseradish peroxidase (Boster, China) and stained with 3'3-diaminobenzidine. For the negative controls, the primary antibody was replaced with non-specific rabbit immunoglobulin G (Cell Signalling Technology, Inc., Beverly, MA, USA). Two pathologists scored the results independently based on the intensity of staining and the extent of expression. The intensity of staining was scored as: 0 , negative; 1 , weak; or 2 , strong. The extent of expression was scored based on the percentage of positive cells: 0 , negative; $1,1-25 \% ; 2,26-50 \%$; $3,51-75 \%$; and $4,76-100 \%$. The overall score of each case was a sum of the intensity of staining and the extent of expression scores. Overall scores of $\geq 4$ were defined as high staining, whereas those $<4$ represented low staining.

Statistical analysis. The SPSS version 15.0 software package (SPSS, Inc., Chicago, IL, USA) was used for statistical analysis. Protein expression versus clinicopathological criteria was assessed using the Pearson $\chi^{2}$ test of association. A logistic regression analysis was used to identify significant multivariate predictors. A multivariate model was used to analyze all the variables that were significant in the univariate analysis. The Kaplan-Meier method was used to calculate the cumulative survival (DFS and OS) time and was analyzed by the log-rank test. The multivariate survival analysis was performed according to the Cox proportional hazards model. $\mathrm{P}<0.05$ was used to indicate a statistically significant difference.

\section{Results}

Expression and correlation of Smac and survivin in pre-treatment biopsies and post-operative specimens. Figure 1 shows representative images of Smac and survivin expression prior to and following NAC. Smac and survivin were mainly detected in the cytoplasm of breast cancer cells. Smac was expressed in $40.8 \%(n=40 / 98)$ of biopsies and in $59.5 \%(n=47 / 79)$ of the resected specimens. Smac expression was significantly upregulated following NAC ( $\mathrm{P}=0.013$; Fig. 1A and B). However, survivin was expressed in $53.1 \%(\mathrm{n}=52 / 98)$ of biopsies and in $36.7 \%(29 / 79)$ of the resected specimens. The expression of survivin was significantly decreased $(\mathrm{P}=0.03)$ in the resected specimens (Fig. 1C and D). Moreover, in the biopsy and surgical specimens, Smac expression was inversely correlated 
Table I. Correlation between Smac and survivin expression and clinicopathological characteristics of primary breast cancer patients.

\begin{tabular}{|c|c|c|c|c|c|c|c|}
\hline \multirow[b]{2}{*}{ Characteristics } & \multirow[b]{2}{*}{ Number } & \multicolumn{2}{|c|}{ Smac, $\mathrm{n}$} & \multirow[b]{2}{*}{ P-value } & \multicolumn{2}{|c|}{ Survivin, $\mathrm{n}$} & \multirow[b]{2}{*}{ P-value } \\
\hline & & High & Low & & High & Low & \\
\hline \multicolumn{8}{|l|}{ Age, years } \\
\hline$\leq 50$ & 46 & 22 & 24 & \multirow[t]{2}{*}{0.184} & 28 & 18 & \multirow[t]{2}{*}{0.145} \\
\hline$>50$ & 52 & 18 & 34 & & 24 & 28 & \\
\hline \multicolumn{8}{|l|}{ Tumor size, $\mathrm{cm}$} \\
\hline$\leq 3$ & 38 & 15 & 23 & \multirow[t]{2}{*}{0.830} & 18 & 20 & \multirow[t]{2}{*}{0.369} \\
\hline$>3$ & 60 & 25 & 35 & & 34 & 26 & \\
\hline \multicolumn{8}{|l|}{ Grade } \\
\hline $\mathrm{I} / \mathrm{II}$ & 45 & 21 & 24 & \multirow[t]{2}{*}{0.278} & 24 & 21 & \multirow[t]{2}{*}{0.960} \\
\hline III & 53 & 19 & 34 & & 28 & 25 & \\
\hline \multicolumn{8}{|c|}{ Lymph node metastasis } \\
\hline Positive & 62 & 26 & 36 & \multirow[t]{2}{*}{0.809} & 34 & 28 & \multirow[t]{2}{*}{0.644} \\
\hline Negative & 36 & 16 & 20 & & 18 & 18 & \\
\hline \multicolumn{8}{|l|}{ TNM stage } \\
\hline II & 43 & 21 & 22 & \multirow[t]{2}{*}{0.153} & 19 & 24 & \multirow[t]{2}{*}{0.120} \\
\hline III & 55 & 19 & 36 & & 33 & 22 & \\
\hline \multicolumn{8}{|l|}{ ER } \\
\hline Positive & 56 & 19 & 37 & \multirow[t]{2}{*}{0.109} & 32 & 24 & \multirow[t]{2}{*}{0.350} \\
\hline Negative & 42 & 21 & 21 & & 20 & 22 & \\
\hline \multicolumn{8}{|l|}{ PR } \\
\hline Positive & 35 & 13 & 22 & \multirow[t]{2}{*}{0.511} & 22 & 13 & \multirow[t]{2}{*}{0.148} \\
\hline Negative & 63 & 27 & 36 & & 30 & 33 & \\
\hline \multicolumn{8}{|l|}{ Her-2 } \\
\hline Positive & 42 & 15 & 27 & \multirow[t]{2}{*}{0.113} & 21 & 21 & \multirow[t]{2}{*}{0.599} \\
\hline Negative & 56 & 25 & 31 & & 31 & 25 & \\
\hline
\end{tabular}

TNM, tumor node metastiasis; ER, estrogen receptor; PR, progesterone receptor; Her-2, human epidermal growth factor receptor 2.

with survivin expression $(\mathrm{r}=-0.217, \mathrm{P}=0.032$; and $\mathrm{r}=-0.335$, $\mathrm{P}=0.003$, respectively).

Correlation between Smac and survivin expression in pre-treatment biopsies and clinicopathological characteristics. Table I shows the full clinicopathological characteristics of the patient cohort that were assessable for Smac and survivin expression. No correlation was observed between the expression levels of survivin and Smac and the clinical factors, including age, tumor size, grade, lymph node metastasis, clinical stage and ER, PR and human epidermal growth factor receptor (Her)-2 expression $(\mathrm{P}>0.05)$.

Smac and survivin protein expression and tumor response. Following anthracycline-based neoadjuvant chemotherapy, 19 patients achieved pCR (19.4\%), and RD was identified in 79 patients $(80.6 \%)$. Various clinicopathological parameters, together with Smac and survivin expression, were subjected to univariate and multivariate analyses for their association with pCR (Table II). Univariate analysis indicated that a lack of ER $(\mathrm{P}=0.004)$, positive Smac $(\mathrm{P}=0.001)$ and negative survivin $(\mathrm{P}=0.037)$ were significantly associated with pCR (Fig. 2A).
On multivariate analysis, ER, Smac and survivin remained significantly associated with $\mathrm{pCR}(\mathrm{P}=0.001, \mathrm{P}=0.031$ and $\mathrm{P}=0.012$, respectively). Smac and survivin were significantly associated with $\mathrm{pCR}$ by multivariate analysis; therefore, their combination was evaluated, as shown in Figure 2B. Breast tumors with no survivin expression and positive Smac expression demonstrated the highest pCR rate (41.67\%), and those with positive survivin expression and a lack of Smac expression revealed the lowest rate $(5.56 \%)$. The breast tumors with negative Smac and negative survivin and those with positive Smac and positive survivin expression demonstrated intermediate pCR rates (13.64 and 25.0\%, respectively).

Smac and survivin protein expression and survival. An analysis of the impact of Smac and survivin status is shown in Figure 3; high expression of Smac in the biopsy specimens was revealed to be a favorable prognostic factor, as measured by OS ( $\mathrm{P}=0.033)$ and DFS ( $\mathrm{P}=0.012)$ (Fig. $3 \mathrm{~A}$ and $\mathrm{B})$. By contrast, the patients with positive survivin expression tended to have a poorer prognosis than the patients with negative survivin expression, as measured by OS $(\mathrm{P}=0.01)$ and $\mathrm{DFS}$ $(\mathrm{P}=0.020)$ (Fig. 3C and D). 
Table II. Univariate and multivariate analyses (logistic regression) for tumor response.

\begin{tabular}{|c|c|c|c|c|c|c|}
\hline \multirow[b]{2}{*}{ Factors } & \multicolumn{2}{|c|}{ Univariate analysis } & \multirow[b]{2}{*}{ P-value } & \multicolumn{2}{|c|}{ Multivariate analysis } & \multirow[b]{2}{*}{ P-value } \\
\hline & OR & $95 \% \mathrm{CI}$ & & OR & $95 \% \mathrm{CI}$ & \\
\hline Age, $\leq 50$ vs. $>50$ years & 1.149 & $0.471-2.799$ & 0.760 & - & - & - \\
\hline Tumor size, $\leq 3$ vs. $>3 \mathrm{~cm}$ & 1.107 & $0.444-2.763$ & 0.828 & - & - & - \\
\hline Node status, $\mathrm{cN}^{-}$vs. $\mathrm{cN}^{+}$ & 0.994 & $0.352-2.808$ & 0.991 & - & - & - \\
\hline Tumor grade, I-II vs. III & 2.112 & $0.730-6.117$ & 0.168 & - & - & - \\
\hline TNM stage, II vs. III & 1.435 & $0.511-4.028$ & 0.493 & - & - & - \\
\hline ER, - vs. + & 5.102 & $1.664-15.625$ & 0.004 & 12.502 & $3.012-52.630$ & 0.001 \\
\hline PR, - vs. + & 1.401 & $0.504-3.894$ & 0.518 & - & - & - \\
\hline Her-2, - vs. + & 1.255 & $0.459-3.429$ & 0.658 & - & - & - \\
\hline Smac, high vs. low & 3.548 & $1.406-8.955$ & 0.001 & 4.141 & $1.142-15.013$ & 0.031 \\
\hline Survivin, low vs. high & 0.326 & $0.128-0.826$ & 0.037 & 5.714 & $1.466-22.222$ & 0.012 \\
\hline
\end{tabular}

95\% CI, 95\% confidence interval; OR, odds ratio; cN, clinical node status; ER, estrogen receptor; PR, progesterone receptor; Her-2, human epidermal growth factor receptor 2; Smac, second mitochondria-derived activator of caspases.

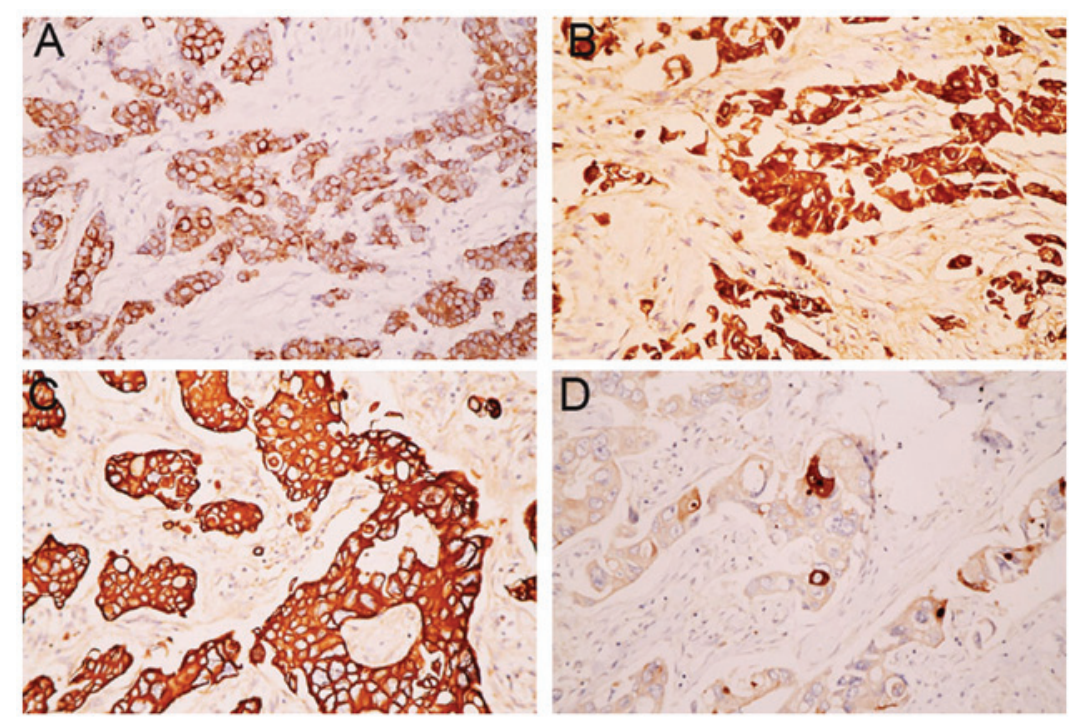

Figure 1. (A) Low expression of Smac in a pre-treatment biopsy. (B) High expression of Smac in a surgical specimen. (C) High expression of survivin in a pretreatment biopsy. (D) Low expression of survivin in a surgical specimen (magnification, $\mathrm{x} 400$ ). Smac, second mitochondria-derived activator of caspases.

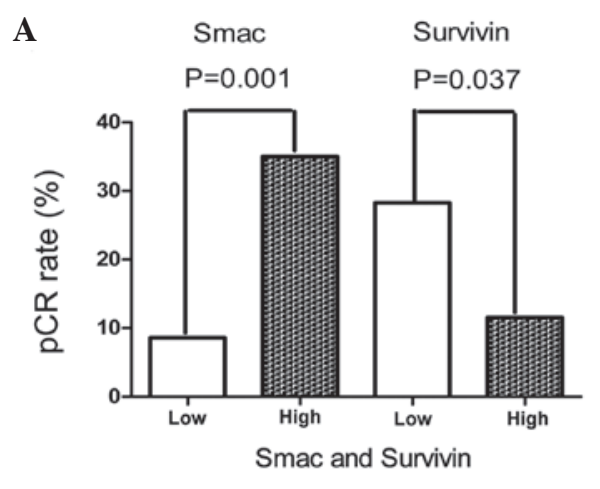

B

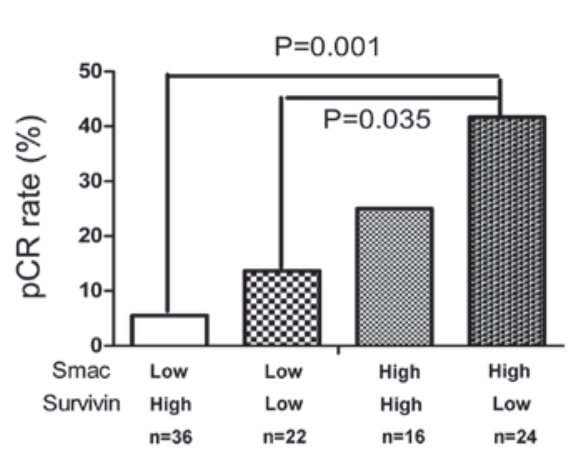

Figure 2. pCR in relation to expression of Smac and survivin in breast tumors prior to NAC. (A) Breast tumors were classified into low and high categories according to the immunostaining results for Smac and survivin prior to NAC. pCR rates are shown for Smac and survivin. (B) pCR rates are shown for the combination of Smac and survivin. pCR, pathological complete response; SMAC, second mitochondria-derived activator of caspases; NAC, neoadjuvant chemotherapy. 
Table III. Multivariate Cox-regression analysis for OS and DFS.

\begin{tabular}{|c|c|c|c|c|c|c|}
\hline \multirow[b]{2}{*}{ Factors } & \multicolumn{2}{|c|}{ OS } & \multirow[b]{2}{*}{ P-value } & \multicolumn{2}{|c|}{ DFS } & \multirow[b]{2}{*}{$\mathrm{P}$-value } \\
\hline & HR & $95 \% \mathrm{CI}$ & & HR & $95 \% \mathrm{CI}$ & \\
\hline Age, $\leq 50$ vs. $>50$ years & 0.621 & $0.272-3.933$ & 0.613 & 0.473 & $0.105-2.132$ & 0.330 \\
\hline Tumor size, $>3$ vs. $\leq 3 \mathrm{~cm}$ & 1.022 & $0.196-8.416$ & 0.485 & 3.145 & $0.469-11.739$ & 0.235 \\
\hline Node status, $\mathrm{cN}^{-}$vs. $\mathrm{cN}^{+}$ & 6.993 & $0.833-18.825$ & 0.073 & 3.362 & $0.549-12.602$ & 0.190 \\
\hline Tumor grade, III vs. I-II & 1.230 & $0.678-4.414$ & 0.252 & 1.010 & $0.247-3.977$ & 0.891 \\
\hline TNM stage, III vs. I-II & 9.497 & $1.491-21.490$ & 0.017 & 4.020 & $1.083-14.929$ & 0.038 \\
\hline ER, - vs. + & 1.323 & $0.531-3.300$ & 0.548 & 1.159 & $0.154-8.735$ & 0.786 \\
\hline PR, - vs. + & 1.247 & $0.475-3.275$ & 0.654 & 2.710 & $0.505-12.492$ & 0.245 \\
\hline Her-2, - vs. + & 1.171 & $0.465-2.950$ & 0.738 & 1.021 & $0.303-3.273$ & 0.895 \\
\hline Smac, low vs. high & 2.950 & $1.173-20.812$ & 0.029 & 3.831 & $1.042-12.085$ & 0.044 \\
\hline Survivin, low vs. high & 8.850 & $1.587-32.682$ & 0.015 & 4.690 & $1.511-14.556$ & 0.007 \\
\hline
\end{tabular}

OS, overall survival; DFS, disease-free survival; 95\% CI, 95\% confidence interval; HR, hazard ratio; cN, clinical node status; ER, estrogen receptor; PR, progesterone receptor; Her-2, human epidermal growth factor recptor 2; Smac, second mitochondria-derived activator of caspases.

A

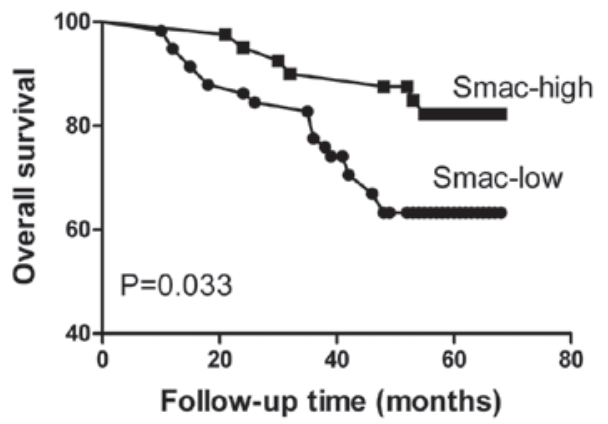

C

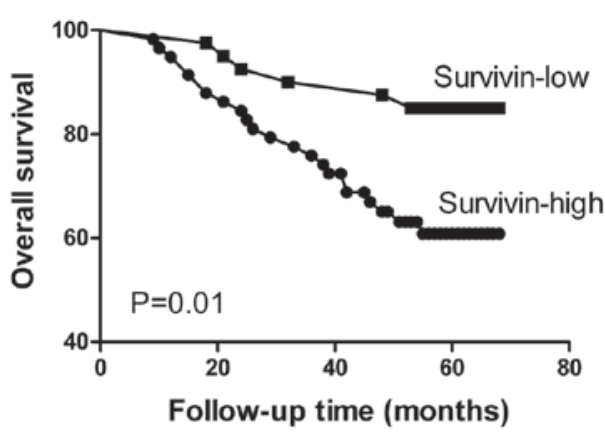

B

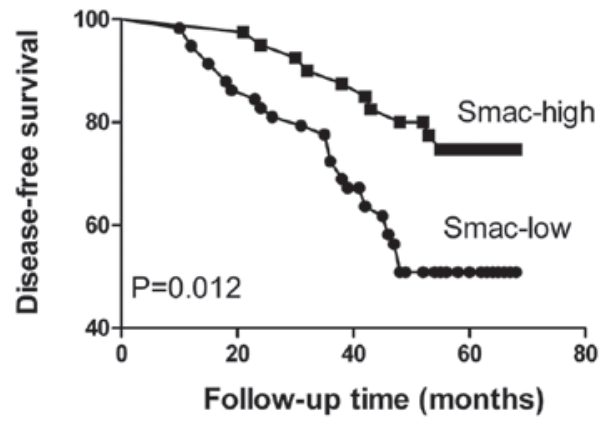

D

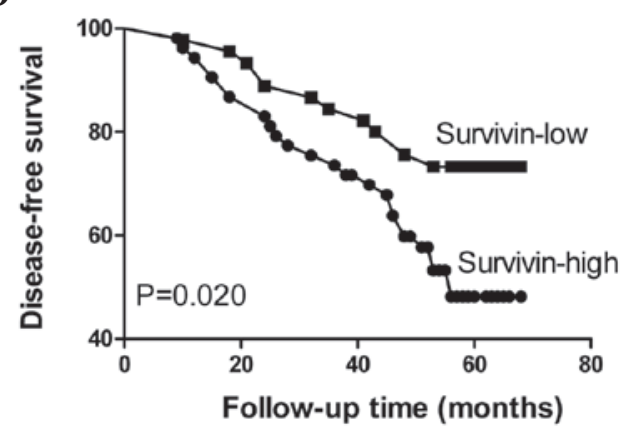

Figure 3. Prognostic significance of biomarkers prior to neoadjuvant chemotherapy in breast cancer. Kaplan-Meier curves for OS and DFS were stratified by low or high subset. Smac demonstrated prognostic significance for (A) OS and (B) DFS. Significant association of high survivin expression with reduced (C) OS and (D) DFS was also identified. OS, overall survival; DFS, disease-free survival; Smac, second mitochondria-derived activator of caspases.

In Cox regression, when analyzing patient age, lymph node metastasis, histological grade, tumor size, TNM stage, Smac expression, survivin expression, ER, PR and Her-2, only Smac expression ( $\mathrm{P}=0.029$; hazard ratio $(\mathrm{HR}), 2.950$; $95 \%$ confidence interval (CI), 1.173-20.812 and $\mathrm{P}=0.044$; HR, 3.831; 95\% CI, 1.042-12.085), survivin expression $(\mathrm{P}=0.015$; HR, 8.850; 95\% CI, 1.587-32.682 and $\mathrm{P}=0.007$; HR, 4.690; 95\% CI, 1.511-14.556) and TNM stage $(\mathrm{P}=0.017$; HR, 9.497, 95\% CI, 1.491-21.490 and $\mathrm{P}=0.038$; HR, 4.020; 95\% CI, 1.083-14.929) remained as independent prognostic factors for OS and DFS, respectively (Table III).

\section{Discussion}

The achievement of a pCR is a significant goal for NAC. Despite intensive efforts to determine the correct regimen for individual breast cancer patients, there is no reliable marker to select the best treatment or to monitor response during therapy. 
To the best of our knowledge, the present study is the first to analyze the potential value of Smac and survivin protein expression in predicting the response of breast cancer to NAC.

Smac may be considered a therapeutic target due to its role as an IAP antagonist. Smac contains a mitochondrial targeting signal that is required for translocation to the cytosol. Upon cleavage of the targeting signal, Smac may enter the cytosol where it may bind to the bacculovirus IAP repeat domain of the IAPs (9). Smac, caspase-3 and -9 are competitive substrates of IAPs, therefore, upon binding of Smac the caspases are released and their substrates cleaved, which as a consequence induces apoptosis. This is known to be significant for normal development and for chemotherapy and radiotherapy responsiveness. Previous studies have indicated that Smac-mediated apoptosis is significant for the apoptotic responses induced by several anticancer agents, including certain chemopreventive agents $(10,11)$. Xu et al indicated that the upregulation of Smac is a chemosensitization mechanism in esophageal squamous cell carcinoma. Smac-knockdown significantly suppressed cisplatin-induced apoptosis, mitochondrial membrane potential collapse, caspase activation and cytochrome $c$ release, leading to cisplatin resistance in vitro and in vivo (12). Fandy et al (13) transiently transfected full-length or mature Smac into breast cancer cells and treated them with anticancer drugs. It was observed that Smac enhanced the antiproliferative and pro-apoptotic effects in MCF-7 cells. In ovarian carcinoma cell lines, infection with an adenovirus encoding Smac increased the sensitivity to chemotherapy and radiotherapy, and increased the activation of caspase-3 and -9 (14). Similarly, the present study revealed that breast cancer patients with high Smac expression had a good response to NAC. Moreover, a high expression of Smac was demonstrated to correlate with improved DFS and OS in patients with advanced breast cancer treated with anthracycline-based chemotherapy. These findings are in line with a study reported by Mizutani et al (15), where renal cell carcinoma patients with positive Smac expression had a longer post-operative disease-specific survival when compared with those with no Smac expression. These results are not unexpected, since overexpression of Smac sensitizes tumor cells against anticancer drug-induced apoptosis (16).

The survivin gene, BIRC 5, is a member of the IAP family and has several cellular functions, including the inhibition of apoptosis and the dysregulation of mitosis, cell cycle progression, carcinogenesis and DNA repair (17). As an IAP, high levels of survivin have been associated with a poor prognosis in various types of human cancer. However, several studies that attempted to delineate the clinical role of survivin expression demonstrated contradictory results with regard to its subcellular localization and prognostic significance $(18,19)$. Certain studies indicated that high levels of cytoplasmic survivin and low levels of nuclear survivin are associated with a poor clinical outcome, whereas others indicated the opposite or reported no correlation $(20,21)$. These discrepancies may reflect differences in the methods used to detect survivin, the different antibodies with differing sensitivities used in immunohistochemistry analyses and the different types of patients selected in the studies. In the present study, survivin was revealed to be most frequently expressed in the cytoplasm, which was concurrent with the study by Span et al (22), which stated that the cytoplasmic form is the predominant cellular species of survivin. Overexpression of survivin is associated with resistance to chemotherapy, and decreased survivin expression is associated with increased sensitivity to etopside and 5-FU (23). In the present study, survivin expression was significantly negatively associated with the response to chemotherapy and with DFS and OS; however, no significant correlation was identified with other clinicopathological factors, including clinical stage, age, grade or lymph node metastasis. The results indicated that high survivin expression in the tumor is predictive of a poorer response and prognosis in patients with advanced breast cancer treated with anthracycline-based NAC. Boidot et al (24) also demonstrated that increased survivin transcript expression is significantly correlated with resistance to an epirubicin-based combination regimen and reduced DFS. However, Estevez et al (25) identified no correlation between nuclear survivin expression and the response to NAC in patients with breast cancer. Thus, future studies evaluating the predictive role of survivin should include a thorough assessment of the expression of different transcripts combined with ultrastructural analyses to clarify whether a cytosolic or nuclear accumulation is a better predictor of response.

In the present study, a significant negative correlation was revealed between the expression of survivin and Smac in the pre-treatment of breast carcinoma $(\mathrm{P}<0.01)$. Following NAC, tumoral Smac expression was upregulated and survivin expression was downregulated. Smac expression also had an inverse correlation with survivin expression. This increase in Smac expression may directly or indirectly result in the downregulation of survivin expression in the sample analyzed, which may explain the increased cell apoptosis during treatment with anticancer agents. Furthermore, Smac-positive and survivin-negative tumors were observed to demonstrate the highest pCR rate. These results confirmed that Smac is an antagonist of survivin, preventing the inhibition of caspases to sensitize cancer cells to NAC.

The growth, differentiation and survival of mammary epithelial cells is directed by a synergy between estrogen and progesterone. The response to hormonal therapy may be predicted by the status of the aforementioned hormones. However, the tumor response to chemotherapy and their role in its prediction remains unclear. Darb-Esfahani et al (26) reported that hormone receptor ${ }^{+} / \mathrm{HER} 2^{+}$-coexpressing carcinomas tend to respond well to NAC, indicating favorable prognoses, and that hormone receptor ${ }^{+} / \mathrm{HER}^{-}$tumors have good prognoses, irrespective of pCR. Similarly, in a retrospective study that included 1,118 patients treated with various NAC regimes, Liedtke et al (27) identified equally high pCR rates in $\mathrm{ER}^{+} / \mathrm{HER}^{+}$and $\mathrm{ER}^{-} / \mathrm{HER} 2^{-}$carcinomas (21 vs. $22 \%$ ). By contrast, two previous clinical studies revealed a correlation between chemosensitivity and a negative hormonal receptor status $(28,29)$. In the present study, the pCR rate of ER-positive tumors was revealed to be significantly lower compared with that of ER-negative tumors. Multivariate analysis demonstrated that an ER-negative status was also a significant predictor for a good response to NAC. One hypothesis states that ER may be a marker of the differentiation of luminal epithelial cells and that well-differentiated tumors are not as likely to be responsive to chemotherapy compared with those that are less differentiated. Furthermore, no statistically significant correlation was 
observed between hormone receptor expression and Smac or survivin in breast cancer. The present observations agree with studies reported by Kennedy et al (30) and Xie et al (31), which demonstrated that no statistically significant correlation exists between survivin and Smac expression and either ER or PR.

In conclusion, among primary breast cancer patients receiving anthracycline-based NAC, patients with positive Smac expression and a lack of survivin expression were more likely to achieve pCR. By assessing the expression of Smac and survivin, the prognosis of breast cancer patients receiving NAC may also be estimated. However, in recent years, taxanes as a standard treatment have been included in the majority of the neoadjuvant study protocols. Furthermore, targeted therapies, including lapatinib, trastuzumab, bevacizumab, pertuzumab, everolimus and others, should be tested, particularly when resistance against a standard chemotherapy is observed or anticipated. Therefore, this may represent a limitation for interpreting the results with regard to the clinical setting. Moreover, due to the retrospective evaluation and the limited sample size, the results should be confirmed in larger cohorts, preferentially in prospective trials.

\section{References}

1. Goldhirsch A, Wood WC, Gelber RD, Coates AS, Thürlimann B and Senn HJ; 10th St. Gallen conference: Progress and promise: highlights of the international expert consensus on the primary therapy of early breast cancer 2007. Ann Oncol 18: 1133-1144, 2007.

2. Kaufmann M, Hortobagyi GN, Goldhirsch A, et al: Recommendations from an international expert panel on the use of neoadjuvant (primary) systemic treatment of operable breast cancer: an update. J Clin Oncol 24: 1940-1949, 2006.

3. Du C, Fang M, Li Y, Li L and Wang X: Smac, a mitochondrial protein that promotes cytochrome c-dependent caspase activation by eliminating IAP inhibition. Cell 102: 33-42, 2000.

4. Verhagen AM, Ekert PG, Pakusch M, et al: Identification of DIABLO, a mammalian protein that promotes apoptosis by binding to and antagonizing IAP proteins. Cell 102: 43-53, 2000.

5. Goyal L: Cell death inhibition: keeping caspases in check. Cell 104: 805-808, 2001.

6. Song Z, Yao X and Wu M: Direct interaction between survivin and Smac/DIABLO is essential for the anti-apoptotic activity of survivin during taxol-induced apoptosis. J Biol Chem 278: 23130-23140, 2003.

7. Sobin LH and Wittekind C (eds): TNM classification of malignant tumours. 6th edition. Wiley-Liss, New York, NY, pp22-26, 2002.

8. Mazouni C, Peintinger F, Wan-Kau S, et al: Residual ductal carcinoma in situ in patients with complete eradication of invasive breast cancer after neoadjuvant chemotherapy does not adversely affect patient outcome. J Clin Oncol 25: 2650-2655, 2007.

9. Martinez-Ruiz G, Maldonado V, Ceballos-Cancino G, Grajeda JP and Melendez-Zajgla J: Role of Smac/DIABLO in cancer progression. J Exp Clin Cancer Res 27: 48, 2008.

10. Bank A, Wang P, Du C, Yu J and Zhang L: SMAC mimetics sensitize nonsteroidal anti-inflammatory drug-induced apoptosis by promoting caspase-3-mediated cytochrome c release. Cancer Res 68: 276-284, 2008.

11. Sun Q, Zheng X, Zhang L and Yu J: Smac modulates chemosensitivity in head and neck cancer cells through the mitochondrial apoptotic pathway. Clin Cancer Res 17: 2361-2372, 2011.

12. Xu Y, Zhou L, Huang J, et al: Role of Smac in determining the chemotherapeutic response of esophageal squamous cell carcinoma. Clin Cancer Res 17: 5412-5422, 2011.
13. Fandy TE, Shankar S and Srivastava RK: Smac/DIABLO enhances the therapeutic potential of chemotherapeutic drugs and irradiation, and sensitizes TRAIL-resistant breast cancer cells. Mol Cancer 7: 60, 2008.

14. McNeish IA, Bell S, McKay T, Tenev T, Marani M and Lemoine NR: Expression of Smac/DIABLO in ovarian carcinoma cells induces apoptosis via a caspase-9-mediated pathway. Exp Cell Res 286: 186-198, 2003.

15. Mizutani Y, Nakanishi H, Yamamoto K, et al: Downregulation of Smac/DIABLO expression in renal cell carcinoma and its prognostic significance. J Clin Oncol 23: 448-454, 2005.

16. Fulda S, Wick W, Weller M and Debatin KM: Smac agonists sensitize for Apo2L/TRAIL- or anticancer drug-induced apoptosis and induce regression of malignant glioma in vivo. Nat Med 8: 808-815, 2002.

17. Blanc-Brude OP, Mesri M, Wall NR, Plescia J, Dohi T and Altieri DC: Therapeutic targeting of the survivin pathway in cancer: initiation of mitochondrial apoptosis and suppression of tumor-associated angiogenesis. Clin Cancer Res 9: 2683-2692, 2003.

18. Shinohara ET, Gonzalez A, Massion PP, et al: Nuclear survivin predicts recurrence and poor survival in patients with resected nonsmall cell lung carcinoma. Cancer 103: 1685-1692, 2005.

19. Falleni M, Pellegrini C, Marchetti A, et al: Survivin gene expression in early-stage non-small cell lung cancer. J Pathol 200: 620-626, 2003.

20. Rexhepaj E, Jirstrom K, O'Connor DP, et al: Validation of cytoplasmic-to-nuclear ratio of survivin as an indicator of improved prognosis in breast cancer. BMC Cancer 10: 639, 2010.

21. Chu JS, Shew JY and Huang CS: Immunohistochemical analysis of survivin expression in primary breast cancers. J Formos Med Assoc 103: 925-931, 2004.

22. Span PN, Sweep FC, Wiegerinck ET, et al: Survivin is an independent prognostic marker for risk stratification of breast cancer patients. Clin Chem 50: 1986-1993, 2004

23. Hayashi N, Asano K, Suzuki H, et al: Adenoviral infection of survivin antisense sensitizes prostate cancer cells to etoposide in vivo. Prostate 65: 10-19, 2005.

24. Boidot R, Vegran F and Lizard-Nacol S: Predictive value of survivin alternative transcript expression in locally advanced breast cancer patients treated with neoadjuvant chemotherapy. Int J Mol Med 23: 285-291, 2009.

25. G Estevez L, Fortes JL, Adrover E, et al: Doxorubicin and cyclophosphamide followed by weekly docetaxel as neoadjuvant treatment of early breast cancer: analysis of biological markers in a GEICAM phase II study. Clin Transl Oncol 11: 54-59, 2009.

26. Darb-Esfahani S, Loibl S, Müller BM, et al: Identification of biology-based breast cancer types with distinct predictive and prognostic features: role of steroid hormone and HER2 receptor expression in patients treated with neoadjuvant anthracycline/taxane-based chemotherapy. Breast Cancer Res 11: R69, 2009.

27. Liedtke C, Mazouni C, Hess KR, et al: Response to neoadjuvant therapy and long-term survival in patients with triple-negative breast cancer. J Clin Oncol 26: 1275-1281, 2008

28. Bear HD, Anderson S, Brown A, et al: The effect on tumor response of adding sequential preoperative docetaxel to preoperative doxorubicin and cyclophosphamide: preliminary results from National Surgical Adjuvant Breast and Bowel Project Protocol B-27. J Clin Oncol 21: 4165-4174, 2003.

29. Colleoni M, Viale G, Zahrieh D, et al: Chemotherapy is more effective in patients with breast cancer not expressing steroid hormone receptors: a study of preoperative treatment. Clin Cancer Res 10: 6622-6628, 2004.

30. Kennedy SM, O'Driscoll L, Purcell R, et al: Prognostic importance of survivin in breast cancer. Br J Cancer 88: 1077-1083, 2003.

31. Xie W, Jiang P, Miao L, et al: Novel link between E2F1 and Smac/DIABLO: proapoptotic Smac/DIABLO is transcriptionally upregulated by E2F1. Nucleic Acids Res 34: 2046-2055, 2006. 